\title{
一种胶状分散体体系下共价有机框架的合成方法
}

刘宗航

济南大学化学化工学院, 济南 250022

E-mail: 201830221241@mail.ujn.edu.cn

\section{Synthesis method of covalent organic frameworks under colloidal dispersion system}

\section{Zonghang Liu}

School of Chemistry and Chemical Engineering, University of Jinan, Jinan 250022, China E-mail: 201830221241@mail.ujn.edu.cn doi: 10.1360/TB-2019-0820

自 2005 年 Yaghi 首次合成具有嗍酸酯基的共价有机 框架(COF-1 和 COF-5)以来 ${ }^{[1]}$, 合成具有可调控性和单晶 结构的共价有机框架材料一直是高分子材料中极具挑战 的课题. 传统的合成方案主要有两步生长法 ${ }^{[2]}$ 和胶体晶核 生长法 ${ }^{[3]}$. 两步生长法对于溶剂和反应物添加速度有着严 格的要求, 而且其反应时间较长, 操作较为繁琐, 难以合 成三聚(自聚)的单晶材料. 胶体晶核生长法可以合成具有 胶体结构前驱液的单晶材料, 但是对溶剂的要求较为严格; 虽然对于二聚 (苯二酚结构与苯嗍酸结构) 有着较好的作 用 ${ }^{[3]}$, 但是一直没有实现三聚反应.

最近, 美国 Northwestern University 的 William R. Dichtel 团队 ${ }^{[4]}$ 工丂妙地用胶体晶核生长法实现了简单苯二嗍 酸结构共价有机框架材料(Ph-COF, BPh-COF, Py-COF, DBD-COF)的合成, 创建了一种硼酸酯基单晶材料的合成 新策略——“聚胶体悬浮液法 (tripoly-colloidal suspensions)”(图 1). 作者不仅介绍了能够合成稳定的二维(2D)、 三维(3D)三硼酸酯共价有机框架的胶体悬浮液法, 还提供 了一种混合溶剂方案. 合成 $\mathrm{Ph}-\mathrm{COF}(\mathrm{COF}-1)$ 时, 在给电子 溶剂体系下 (主要采用乙腈、1,4-二氧六环和均三甲苯, 其 中, 1,4 二氧六环: 均三甲苯 $=4: 1$, 他们明确了特定溶剂乙 腈所占比例: 乙腈占混合溶剂体积分数在 $30 \%$ 以下时, 合 成过程中会出现沉淀; 30\% 80\% 时, 处于胶体悬浮液状态; $80 \%$ 以上时, 溶液呈透明状态.

该研究利用同步辐射 $\mathrm{X}$ 射线衍射仪(SXRD)和高分辨
透射电子显微镜(HR-TEM)进行了分析, 深人了解了这些材 料的层间排列, 并证实了它们的单晶性质. 此外, 作为胶体 悬浮液, 稳定 COF 能够利用苂光激发-发射矩阵光谱(EEMS) 探测这些材料的固有光学性质. 溶液苂光光谱结果表明, 与 它们各自的单体溶液相比, 共价有机框架晶体具有高发射 率. 而激发发射矩阵荧光光谱结果表明, 这种增强发射的起 源可归因于片状结构之间的发色团的空间连通. 该方法融 合了两步生长法和胶体晶核生长法, 首次用胶体晶核生长 法合成了三聚硼酸酯结构; 相较于该团队前期的二聚方

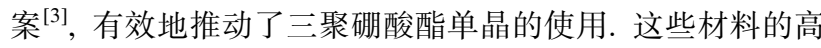
质量光谱结果显示, 我们能够将光学特征的起源归结到 $\pi$ 激 基复合物的形成, 这表明在 COF 结构中紧密靠近的生色团 可以通过空间电子的相互作用, 实现极高的使用价值.

值得指出的是，在二维、三维共价有机框架材料合成 主要以碎晶和粉晶形式进行的情况下, 胶体晶核生长法对 于完整晶体结构共价有机框架材料的合成有着重要意义; 同时, 这种方法也是一种简捷、便宜、高效的硼酸酯基共 价有机框架合成方法. 这些结果表明, 给电子溶剂的存在 对环硼氧烷连接骨架胶体起到了稳定作用. 当然, 该方案 也存在一定的不足：一方面，对于溶剂条件的篮选较少， 无法精确调控乙腈的最佳添加比例; 另一方面, 该方案虽 然操作比较简便, 但反应时间较长, 三聚需要的反应时间 长达 $3 \mathrm{~d}$, 而二聚的合成时间只需要 $18 \mathrm{~h}$. 因此, 如何优化 筛选反应条件是该方法能否推广的重要因素. 
(a)

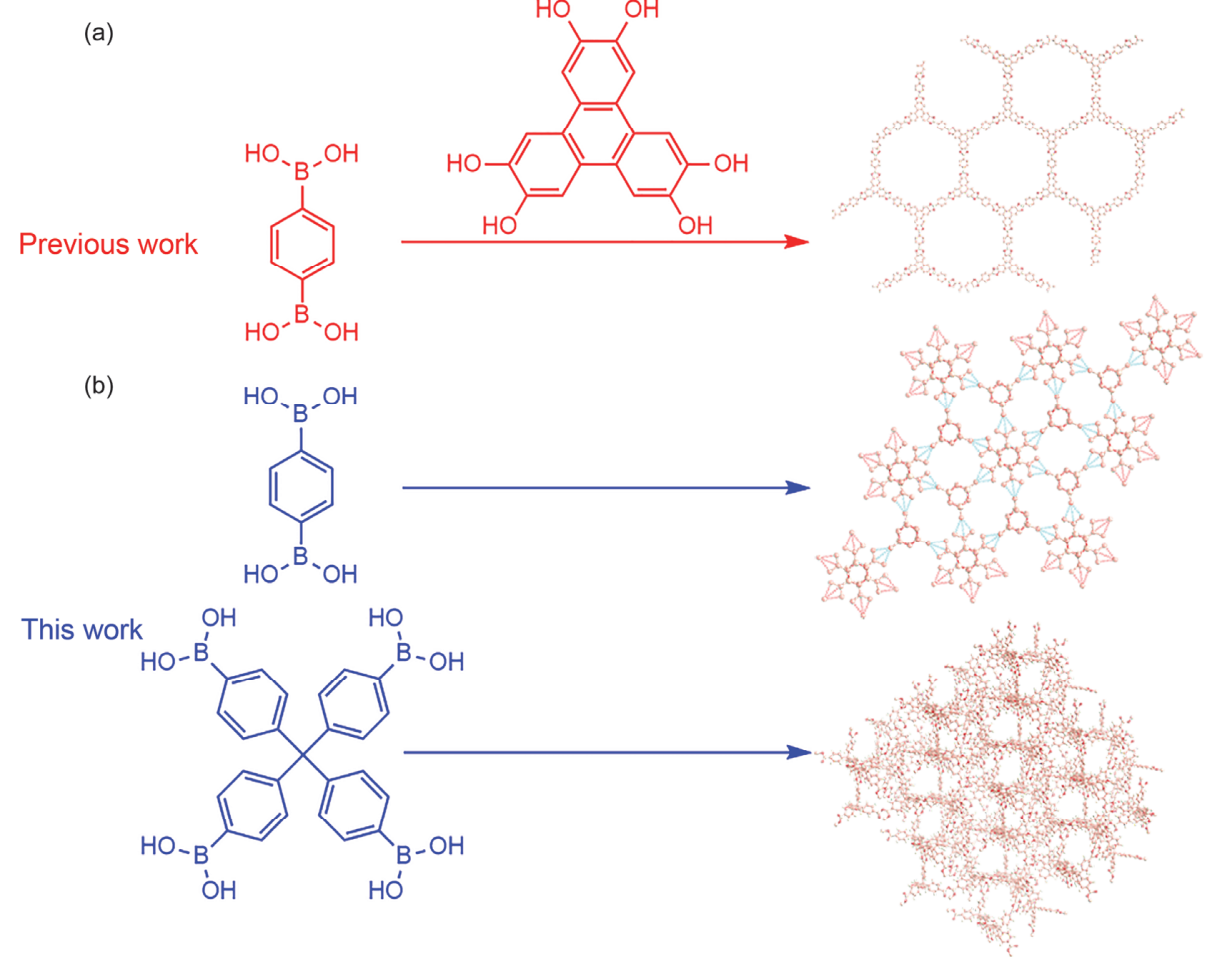

图 1 (网络版彩色)传统合成方法和本方法的示意图. (a) 传统合成方法在胶体悬浮法下合成二嗍酸酯聚合物结构, 并验证了二维硼酸 酯共价有机框架的稳定性; (b) 本方法采用嗍酸自缩合形成的三氧三硼烷结构, 证明了在给电子溶剂下可以实现 $2 \mathrm{D}$ 和 $3 \mathrm{D}$ 共价有机框架 单晶的稳定存在 ${ }^{[4]}$

Figure 1 (Color online) Schematic diagrams of previous work and this work. (a) Previous work: Synthesize the diborate polymer structure under colloidal suspension method and complete the stability verification of 2D borate COFs. (b) This work: The trioxatriborinane structure was formed by the self-condensation of boric acid, and it was proved that the stability of 2D and 3D COFs single crystals can be completed under an electron-donating solvent ${ }^{[4]}$

\section{推荐阅读文献}

1 Cote A P, Benin A I, Ockwig N W, et al. Porous, crystalline, covalent organic frameworks. Science, 2005, 310: 1166-1170

2 Zhang Y B, Su J, Hiroyasu F, et al. Single-crystal structure of a covalent organic framework. J Am Chem Soc, 2013, 135: 16336-16339

3 Austin M E, Lucas R P, Nathan C F, et al. Seeded growth of single-crystal two-dimensional covalent organic frameworks. Science, 2018, 361: $52-57$

4 Austin M E, Ioannina C, Alexandra B, et al. Emissive single-crystalline boroxine-linked colloidal covalent organic frameworks. J Am Chem Soc, 2019, 141: 19728-19735 